ments, which may, in fact, be constructed with widely varying proportions, the information available is not of much use. Also references to the impulse tangent circle are misleading, since impulse is, in fact, delivered at right-angles to these lines, and in most of the drawings of clock and lever watch pallets there are small but nevertheless important geometrical errors.

Gearing is dealt with in Chapter 5, and in the supplement, and an interesting section has been included on epicyclic trains for revolving-escapement watches. There is a very useful section on the balance and spring, covering both the questions of temperature and positional errors; the supplement includes a section on modern hairspring alloys.

A good description of striking and chiming mechanisms, general lay-outs of clocks and watches, and much interesting and useful information on complicated watches, such as chronographs, calendars and repeaters, with a good chapter on the marine chronometer, combine to make this book very useful and informative to those interested in horology from a descriptive point of view.

R. A. FeLI

\section{LABORATORY ORGANIC CHEMISTRY}

Newer Methods of Preparative Organic Chemistry Translated and revised from the German. (Published and distributed in the Public Interest with the consent of the Alien Property Custodian.) Pp. xiii +657. (New York and London: Interscience Publishers, Inc., 1948.) 8.50 dollars; 518.

Semimicro Qualitative Organic Analysis

By Nicholas D. Cheronis and John B. Entrikin. Pp. xiv +498 . (New York: Thomas Y. Crowell Company ; London : Constable and Co., Ltd., 1947.) 25s. net.

\section{Reactions of Organic Compounds}

By Dr. Wilfred John Hickinbottom. Second edition. Pp. $x+482$. (London, New York and Toronto: Longmans, Green and Co., Ltd., 1948.) 25s. net.

$\mathrm{T}$ HESE three books cater for the needs of the student and the worker in organic chemical research.

"Newer Methods of Preparative Organic Chemis. try" comprises fourteen articles each written by a German expert, which have been translated and revised by American chemists. It thus presents the reader with authoritative accounts of the application in the laboratory of a number of the most useful of modern methods and special reagents. Oxidations using lead tetra-acetate or periodic acid and dehydro. genations with sulphur, selenium or platinum and palladium catalysts are described. Catalytic reductions with Raney nickel or copper chromite are reviewed in detail. Another chapter is devoted to oxidations and reductions in presence of aluminium alkoxides. An account of biochemical oxidations and reductions deals with the employment for preparative purposes not only of micro-organisms but also of higher animals.

Other sections of this book discuss various substitution reactions of aliphatic substances, the chemistry of organic fluorine compounds and the uses of boron fluoride and hydrofluoric acid in organic reactions.
The fact that the chapters on diene syntheses and the uses of diazomethane were written by Alder and Eistert respectively is a guarantee of their excellence, and in conclusion an account is given of the uses of organolithium compounds.

The volume is an excellent and timely production which will be of great service to the research worker.

"Semimicro Qualitative Organic Analysis" aims at providing a general course in qualitative organic analysis for students, and a manual and reference book for workers in all types of laboratory, whether academic, professional or industrial.

The rapid expansion of our knowledge of the chemistry of biological materials has been largely due to the use of microchemical methods, the advantages of which are generally recognized, and the research worker in organic chemistry must sooner or later acquire the necessary technique. The principle adopted by the authors of this book is that the student should learn to work on the semimicro scale in the organic laboratory from his first year, and the advantages in economy of material and valuable training are obvious. All the necessary operations and equipment are, therefore, described at the outset, and the rest of the book gives a systematic procedure for the identification of a substance, special emphasis being placed on observations of solubility. Suitable derivatives are listed for each class of compound and exact directions are given for their preparation and careful purification on the semimicro scale.

Numerous lists of selected references are included, and the book concludes with a valuable collection of tables of compounds arranged in classes in order of melting or boiling point together with particulars of various derivatives or figures for physical properties such as density, refractive index or rotatory power to assist the identification. A final table includes many more complex bases, drugs, vitamins and hormones.

This volume will not only be useful to the student, but will also prove most valuable as a book of reference.

A new and revised edition of Hickinbottom's "Reactions of Organic Compounds" will be generally welcomed. It reviews in detail the reactions of all the main classes of compounds and gives in an appendix a scheme for their identification. New material has been added, particularly in the sections on saturated and unsaturated hydrocarbons, on organolithium compounds and on the uses of diazomethane. The book is a valuable source of information with many preparative details, and it is packed with references to the literature.

\section{APPLIED SURFACE CHEMISTRY}

\section{Surface Chemistry for Industrial Research}

By J. J. Bikerman. Pp. ix +464 . (New York: Academic Press, Inc.; London: H. K. Lewis and Co., Ltd., 1948.) 8 dollars.

THE author states in his preface that "This volume is an exposition, adapted to Industrial Research, of the fundamentals of Surface Science". In fact, it is nothing of the sort; it is an assemblage of data of surface phenomena with the fundamentals badly scamped. Too frequently also, the author introduces a topic with a definite statement and then proceeds to cite reservations and evidence against it 